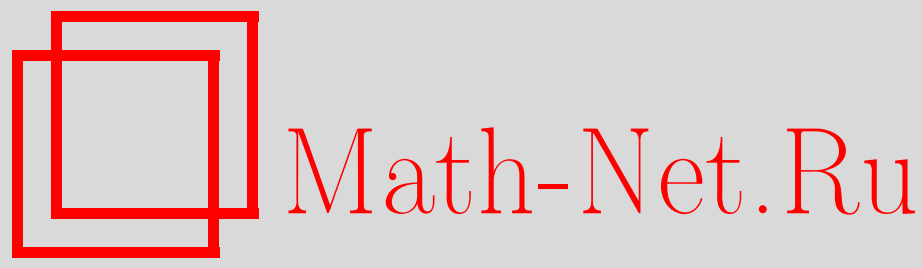

Ю. А. Кашлев, Второе начало термодинамики для высокоэнергетических каналированных частиц, ТМ $\Phi, 2010$, том 162, номер 2, 275-284

DOI: https://doi.org/10.4213/tmf6470

Использование Общероссийского математического портала Math-Net.Ru подразумевает, что вы прочитали и согласны с пользовательским соглашением http://www. mathnet.ru/rus/agreement

Параметры загрузки:

IP : 54.162 .27 .143

26 апреля 2023 г., 12:38:09

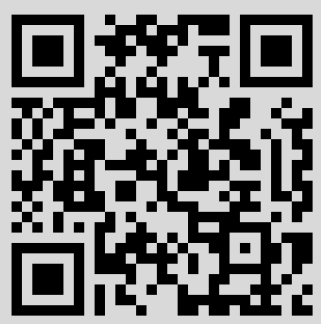




\title{
ФИЗИКА
}

Том 162, № 2

февраль, 2010

2010 г.

\author{
Ю. А. Кашлев*
}

\section{ВТОРОЕ НАЧАЛО ТЕРМОДИНАМИКИ ДЛЯ ВЫСОКОЭНЕРГЕТИЧЕСКИХ КАНАЛИРОВАННЫХ ЧАСТИЦ}

\begin{abstract}
Число кинетических уравнений, на основе которых в рамках микроскопической теории может быть доказано второе начало термодинамики, весьма ограниченно. Доказано выполнение второго начала термодинамики в явном виде для высокоэнергетических частиц, движущихся в кристалле в режиме каналирования. Для доказательства используется локальное уравнение Больцмана для функции распределения частиц, записанное в форме Боголюбова. При этом учитывается один статистический механизм - рассеяние каналированных частиц на атомах решетки, случайным образом сдвинутых из узлов кристалла.
\end{abstract}

Ключевые слова: термодинамика, второй закон (второе начало), каналированная частица, кристалл, кинетическое уравнение Больцмана, тепловое рассеяние, функция распределения, энтропия, энтропийный источник, локальное уравнение баланса энтропии.

\section{1. ОСНОВНЫЕ ОПРЕДЕЛЕНИЯ В СЛУЧАЕ ДВУХКОМПОНЕНТНОЙ СИСТЕМЫ}

Рассмотрим двухкомпонентную систему из быстрых атомных частиц и атомов кристалла. В частности, элемент объема фазового пространства, соответствующего комплексу из одной быстрой частицы и $r$ атомов решетки, можно обозначить $d x_{1}^{\mathrm{g}} d x_{1}^{\mathrm{c}} \ldots d x_{r}^{\mathrm{c}}$, где $x_{1}^{\mathrm{g}}=\left(\mathbf{q}_{1}^{\mathrm{g}}, \mathbf{p}_{1}^{\mathrm{g}}\right), x_{n}^{\mathrm{c}}=\left(\mathbf{q}_{n}^{\mathrm{c}}, \mathbf{p}_{n}^{\mathrm{c}}\right), \mathbf{q}_{1}^{\mathrm{g}}$ и $\mathbf{p}_{1}^{\mathrm{g}}-$ координата и импульс частицы, $\mathbf{q}_{n}^{\mathrm{c}}$ и $\mathbf{p}_{n}^{\mathrm{c}}-$ координата и импульс $n$-го атома, $n=1, \ldots, r$.

Общий метод исследования систем взаимодействующих частиц был развит в монографии [1], где была введена последовательность функций распределения, характеризующих вероятностные распределения группы частиц. Разумеется, при написании распределения указанного комплекса необходимо учитывать многочастичные корреляции в кристалле, поскольку любой атом решетки может вовлекать в движение совокупность большого числа соседних атомов.

Взяв за основу идею монографии [1], будем считать, что функция распределения комплекса $F_{1 r}$ зависит от времени только через временну́ю зависимость одночастичных функций $F_{10}(t)$ и $F_{01}(t)$, а также временну́ю зависимость всех возможных

${ }^{*}$ Институт металлургии и материаловедения им. А. А. Байкова РАН, Москва, Россия 
корреляционных функций атомов решетки. В этом случае имеем

$$
F_{1 r} \equiv F_{1 r}\left(x_{1}^{\mathrm{g}}, x_{1}^{\mathrm{c}}, \ldots, x_{r}^{\mathrm{c}} \mid F_{10}(t), F_{01}(t), \ldots, F_{0 r}(t)\right),
$$

где

$$
\begin{aligned}
& F_{10}(t)=F_{10}\left(x_{1}^{\mathrm{g}}, t\right), \\
& F_{01}(t)=F_{01}\left(x_{1}^{\mathrm{c}}, t\right), \\
& F_{02}(t)=F_{02}\left(x_{1}^{\mathrm{c}}, x_{2}^{\mathrm{c}} \mid F_{01}(t)\right), \\
& \ldots \ldots \ldots \ldots \ldots \ldots \ldots \ldots \ldots \ldots \ldots, \\
& F_{0 r}(t)=F_{0 r}\left(x_{1}^{\mathrm{c}}, \ldots, x_{r}^{\mathrm{c}} \mid F_{01}(t)\right) .
\end{aligned}
$$

Эволюция функций распределения описывается цепочкой уравнений ББГКИ [1]

$$
\begin{gathered}
\frac{\partial}{\partial t} F_{1 r}=\left[H_{1 r} ; F_{1 r}\right]+n_{\mathrm{c}} \int d x_{r+1}^{\mathrm{c}}\left[\Phi_{1, r+1} ; F_{1, r+1}\right]+ \\
+n_{\mathrm{c}} \sum_{1 \leqslant m \leqslant r} \int d x_{r+1}^{\mathrm{c}}\left[\Phi_{m, r+1}^{\mathrm{c}} ; F_{1, r+1}\right], \\
\frac{\partial}{\partial t} F_{0 r}=\left[H_{0 r} ; F_{0 r}\right]+n_{\mathrm{c}} \sum_{1 \leqslant m \leqslant r} \int d x_{r+1}^{\mathrm{c}}\left[\Phi_{m, r+1}^{\mathrm{c}} ; F_{0, r+1}\right],
\end{gathered}
$$

где гамильтониан системы задается равенствами

$$
\begin{aligned}
& H_{0 r}=\sum_{1 \leqslant n \leqslant r} \frac{\left(p_{n}^{\mathrm{c}}\right)^{2}}{2 M}+U_{r}^{\mathrm{c}}, \quad U_{r}^{\mathrm{c}}=\sum_{1 \leqslant m<n \leqslant r} \Phi_{m n}^{\mathrm{c}}, \\
& H_{1 r}=\frac{\left(p_{1}^{\mathrm{g}}\right)^{2}}{2 m}+\sum_{1 \leqslant n \leqslant r} \frac{\left(p_{n}^{\mathrm{c}}\right)^{2}}{2 M}+\sum_{1 \leqslant n \leqslant r} \Phi_{1 n}+U_{r}^{\mathrm{c}},
\end{aligned}
$$

$m$ - масса частицы, $M$ - масса атома решетки, $\Phi_{1 n}$ - потенциал взаимодействия частицы с атомом решетки, $\Phi_{m n}^{c}$ - потенциал взаимодействия атомов решетки между собой, $[A ; B]-$ классические скобки Пуассона, $n_{\mathrm{c}}-$ плотность атомов решетки. При выводе уравнения (2) предполагалось, что взаимодействием между частицами пучка можно пренебречь, поскольку плотность частиц очень мала.

Целью дальнейшего исследования является получение обобщенного кинетического уравнения для функции $F_{10}(t)$, а именно уравнения вида

$$
\frac{\partial}{\partial t} F_{10}(t)=A\left(x_{1}^{\mathrm{g}} \mid F_{10}(t)\right)
$$

Здесь $A\left(x_{1}^{\mathrm{g}} \mid F_{10}(t)\right)$ - функционал, который в любой момент времени $t$ полностью определяется формой распределения $F_{10}(t)$ для того же момента.

В некоторых физических ситуациях цепочка (1), (2) содержит малый параметр, что дает возможность найти решение уравнений в виде разложения по степеням этого параметра [1], [2]. Малый параметр в уравнениях (1), (2) возникает и при анализе движения быстрых частиц, если перейти к безразмерным переменным. Пусть потенциал $\Phi_{1 n}$ имеет короткий радиус действия $r_{0}$, тогда интегральные члены в $(1),(2)$ 
преобразуются таким образом, что основной вклад вносит безразмерная область порядка единицы, при этом в столкновительных членах появляется безразмерный параметр [3]

$$
\beta=n_{\mathrm{c}} r_{0}^{3}\left(\frac{\varepsilon_{0}}{2 E_{0}}\right) \ll 1,
$$

где $E_{0}-$ энергия частицы, $\varepsilon_{0}-$ энергия взаимодействия.

Однако можно использовать более простой подход, избежав преобразований, связанных с введением безразмерных переменных. Для этого достаточно считать $\beta$ формальным параметром малости и ввести его только для того, чтобы дать представление о порядке величины отдельных членов кинетического уравнения. Естественно, в этом случае в окончательных формулах необходимо считать $\beta=1$ [1]. Если разложение распределения по степеням формального параметра

$$
F_{1 r}=F_{1 r}^{(0)}+\beta F_{1 r}^{(1)}+\beta^{2} F_{1 r}^{(2)}+\cdots
$$

подставить в уравнение движения для $F_{1 r}$, то, положив в нем $r=0$, получим искомое уравнение - кинетическое уравнение вида (3).

Сделаем еще одно полезное замечание. Явный вид функции $F_{11}^{(0)}$, вошедшей в разложение (4) при $r=1$, нетрудно получить, зная вид $F_{10}(t)$ и $F_{01}(t)$. Находим

$$
F_{11}^{(0)}\left(x_{1}^{\mathrm{g}}, x_{1}^{\mathrm{c}}, t\right)=F_{10}\left(X_{1}^{\mathrm{g}}\left(x_{1}^{\mathrm{g}}, x_{1}^{\mathrm{c}}\right), t\right) F_{01}\left(X_{1}^{\mathrm{c}}\left(x_{1}^{\mathrm{g}}, x_{1}^{\mathrm{c}}\right), t\right) .
$$

Аргументами функции $F_{10}$ являются динамические переменные атома решетки, взаимодействующего с частицей, и

$$
\begin{aligned}
& X_{k}^{\mathrm{c}}\left(x_{1}^{\mathrm{g}} ; x_{1}^{\mathrm{c}}, \ldots, x_{r}^{\mathrm{c}}\right)=\left\{Q_{k}^{\mathrm{c}}\left(x_{1}^{\mathrm{g}} ; x_{1}^{\mathrm{c}}, \ldots, x_{r}^{\mathrm{c}}\right), P_{k}^{\mathrm{c}}\left(x_{1}^{\mathrm{g}} ; x_{1}^{\mathrm{c}}, \ldots, x_{r}^{\mathrm{c}}\right)\right\}, \\
& Q_{k}^{\mathrm{c}}\left(x_{1}^{\mathrm{g}} ; x_{1}^{\mathrm{c}}, \ldots, x_{r}^{\mathrm{c}}\right)=\lim _{\tau \rightarrow \infty}\left\{S_{-\tau}^{1 r} q_{k}^{\mathrm{c}}+\frac{1}{M} S_{-t}^{1 r} p_{k}^{\mathrm{c}} \tau\right\}, \\
& P_{k}^{\mathrm{c}}\left(x_{1}^{\mathrm{g}} ; x_{1}^{\mathrm{c}}, \ldots, x_{r}^{\mathrm{c}}\right)=\lim _{\tau \rightarrow \infty} S_{-\tau}^{1 r} p_{k}^{\mathrm{c}},
\end{aligned}
$$

где $S_{\tau}^{1 r}$ - оператор сдвига состояния на временной интервал $\tau$ [1]. Аналогично можно определить динамические переменные $X_{k}^{\mathrm{g}}$ быстрой частицы, взаимодействующей с атомами решетки.

\section{2. ЛОКАЛЬНОЕ КИНЕТИЧЕСКОЕ УРАВНЕНИЕ ДЛЯ ФУНКЦИИ РАСПРЕДЕЛЕНИЯ КАНАЛИРОВАННЫХ ЧАСТИЦ. ПЕРЕХОД К ОДНОКОМПОНЕНТНОЙ СИСТЕМЕ}

Рассмотрим особый тип движения заряженных атомных частиц - плоскостное каналирование, при котором частицы совершают устойчивые колебания между стенками - соседними кристаллографическими плоскостями [4], [5] - и движутся поступательно вдоль этих плоскостей. В простейшей модели предполагается, что заряженная частица отражается от положительно заряженной плоскости под тем же углом, под которым она падала на плоскость.

Нетрудно выполнить переход от двухкомпонентной системы, которой соответствуют уравнения (1), (2), к однокомпонентной, если учесть, что в условиях каналирования продольная скорость частицы $v_{0}$ много больше скорости теплового 
движения атомов решетки. Разумеется, в этом случае можно считать, что быстрая частица движется в “замороженной” решетке с фиксированными отклонениями атомов от узлов идеального кристалла.

Если считать, что частица отражается от плоскости под углом настолько малым, что прохождением ее через плоскость (в рамках классической теории) можно полностью пренебречь, то продольная составляющая начального импульса частицы остается постоянной [6]. Изменяется лишь поперечная составляющая $p_{x}$ в результате неупругого рассеяния частицы на фиксированных атомах решетки (ось $x$ перпендикулярна плоскости, образующей стенку канала.) Кроме того, движение частицы вдоль плоскости канала сопровождается большим числом коррелированных взаимодействий, при которых в каждом акте взаимодействия частица незначительно отклоняется от первоначального направления движения (эффект стенки в непрерывном приближении [4]).

Чтобы получить кинетическое уравнение для каналированных частиц в указанной модели, достаточно записать уравнение (1) при $r=0$ и подставить в него разложение (4). Тогда с точностью до членов порядка $\beta^{2}$ получим

$$
\frac{\partial}{\partial t} F_{10}=\left[H_{10} ; F_{10}\right]+\beta n_{\mathrm{c}} \int d x_{1}^{\mathrm{c}}\left[\Phi_{11} ; F_{11}^{(0)}\right]+\beta^{2} n_{\mathrm{c}} \int d x_{1}^{\mathrm{c}}\left[\Phi_{11} ; F_{11}^{(1)}\right] .
$$

Затем следует задать в явном виде переменные (5), вошедшие в (6). Для этого, используя уравнения динамики, вычислим координаты и импульсы частиц после столкновения, аппроксимируя прямыми траектории частиц между двумя последовательными актами рассеяния. В этом приближении получаем

$$
\begin{aligned}
& X_{1}^{\mathrm{g}}=\left\{\widetilde{\mathbf{Q}}_{1}^{\mathrm{g}}, \widetilde{\mathbf{P}}_{1}^{\mathrm{g}}\right\}, \quad X_{1}^{\mathrm{c}}=\left\{\widetilde{\mathbf{Q}}_{1}^{\mathrm{c}}, \widetilde{\mathbf{P}}_{1}^{\mathrm{c}}\right\}, \\
& \widetilde{\mathbf{Q}}_{1}^{\mathrm{g}}=\mathbf{q}_{1}^{\mathrm{g}}+\boldsymbol{\xi}^{\mathrm{g}}, \quad \widetilde{\mathbf{P}}_{1}^{\mathrm{g}}=\mathbf{p}_{1}^{\mathrm{g}}+\boldsymbol{\zeta}^{\mathrm{g}}, \quad \widetilde{\mathbf{Q}}_{1}^{\mathrm{c}}=\mathbf{q}_{1}^{\mathrm{c}}+\boldsymbol{\xi}^{\mathrm{c}}, \quad \widetilde{\mathbf{P}}_{1}^{\mathrm{c}}=\mathbf{p}_{1}^{\mathrm{c}}+\boldsymbol{\zeta}^{\mathrm{c}}, \\
& \boldsymbol{\zeta}^{\mathrm{g}}=\int_{0}^{\infty} d \tau \nabla_{q^{\mathrm{g}}} \Phi\left(\left|\mathbf{q}^{\mathrm{g}}-\mathbf{q}^{\mathrm{c}}-\left(\mathbf{v}^{\mathrm{g}}-\mathbf{v}^{\mathrm{c}}\right) \tau\right|\right), \quad \boldsymbol{\zeta}^{\mathrm{g}}=-\boldsymbol{\zeta}^{\mathrm{c}}, \\
& \boldsymbol{\xi}^{\mathrm{g}}=\frac{1}{m} \int_{0}^{\infty} d \tau \tau \boldsymbol{\nabla}_{q^{\mathrm{g}}} \Phi\left(\left|\mathbf{q}^{\mathrm{g}}-\mathbf{q}^{\mathrm{c}}-\left(\mathbf{v}^{\mathrm{g}}-\mathbf{v}^{\mathrm{c}}\right) \tau\right|\right), \quad \boldsymbol{\xi}^{\mathrm{g}}=-\left(\frac{M}{m}\right) \boldsymbol{\xi}^{c},
\end{aligned}
$$

где $\mathbf{v}^{\mathrm{c}}$ и $\mathbf{v}^{\mathrm{g}}$ - скорости атома решетки и частицы.

Раскрывая классические скобки Пуассона в (6), после ряда преобразований с учетом (5) и (7) находим искомое уравнение - уравнение Больцмана в форме Боголюбова

$$
\begin{gathered}
\frac{\partial}{\partial t} F_{10}\left(x^{\mathrm{g}}, t\right)+\sum_{i} v_{i}^{\mathrm{g}} \frac{\partial}{\partial q_{i}^{\mathrm{g}}} F_{10}\left(x^{\mathrm{g}}, t\right)=\left(\frac{\partial F_{10}}{\partial t}\right)_{\mathrm{st}} \\
\left(\frac{\partial F_{10}}{\partial t}\right)_{\mathrm{st}}=n_{\mathrm{c}} \beta \sum_{i} \int d x_{1}^{\mathrm{c}} \frac{\partial}{\partial q_{i}^{\mathrm{g}}} \Phi\left(\left|\mathbf{q}^{\mathrm{g}}-\mathbf{q}_{1}^{\mathrm{c}}\right|\right) \frac{\partial}{\partial p_{i}^{\mathrm{g}}}\left\{F_{10}\left(\widetilde{\mathbf{Q}}^{\mathrm{g}}, \widetilde{\mathbf{P}}^{\mathrm{g}}, t\right) F_{01}\left(\widetilde{\mathbf{Q}}_{1}^{\mathrm{c}}, \widetilde{\mathbf{P}}_{1}^{\mathrm{c}}\right)\right\}+ \\
+n_{\mathrm{c}}^{2} \beta^{2} \sum_{i, j} \int d x_{1}^{\mathrm{c}} \frac{\partial}{\partial q_{i}^{\mathrm{g}}} \Phi\left(\left|\mathbf{q}^{\mathrm{g}}-\mathbf{q}_{1}^{\mathrm{c}}\right|\right) \times
\end{gathered}
$$




$$
\begin{aligned}
& \times \int d x_{2}^{\mathrm{c}} \frac{\partial}{\partial p_{i}^{\mathrm{g}}}\left\{\frac{\partial}{\partial p_{j}^{\mathrm{g}}} F_{10}\left(x^{\mathrm{g}}, t\right)\left[F_{02}\left(x_{1}^{\mathrm{c}}, x_{2}^{\mathrm{c}}\right)-F_{01}\left(x_{1}^{\mathrm{c}}\right) F_{01}\left(x_{2}^{\mathrm{c}}\right)\right]\right\} \times \\
& \times \int_{0}^{\infty} d \tau \frac{\partial}{\partial q_{j}^{\mathrm{g}}} \Phi\left(\left|\mathbf{q}^{\mathrm{g}}-\mathbf{q}_{2}^{\mathrm{c}}-\left(\mathbf{v}^{\mathrm{g}}-\mathbf{v}_{2}^{\mathrm{c}}\right) \tau\right|\right),
\end{aligned}
$$

где $i, j=1,2,3 ; q_{x}^{\mathrm{g}}=q_{1}^{\mathrm{g}}, q_{y}^{\mathrm{g}}=q_{2}^{\mathrm{g}}, q_{z}^{\mathrm{g}}=q_{3}^{\mathrm{g}}$. Что же касается стационарных функций кристалла $F_{01}$ и $F_{02}$, то, учитывая близость кристалла к состоянию термодинамического равновесия при температуре $T$, эти функции можно представить в виде

$$
\begin{gathered}
n_{\mathrm{c}} F_{01}\left(x^{\mathrm{c}}\right)=\widetilde{F}_{01}\left(q^{\mathrm{c}}\right) \varphi\left(p^{\mathrm{c}}\right), \quad n_{\mathrm{c}}^{2} F_{02}\left(x_{1}^{\mathrm{c}}, x_{2}^{\mathrm{c}}\right)=\widetilde{F}_{02}\left(q_{1}^{\mathrm{c}}, q_{2}^{\mathrm{c}}\right) \varphi\left(p_{1}^{\mathrm{c}}\right) \varphi\left(p_{2}^{\mathrm{c}}\right), \\
\varphi\left(p^{\mathrm{c}}\right)=(2 \pi M T)^{-3 / 2} e^{-\left(p^{\mathrm{c}}\right)^{2} / 2 M T} .
\end{gathered}
$$

Кроме того, следует иметь в виду, что при вычислении столкновительного члена с точностью до членов второго порядка по потенциалу взаимодействия переменные $X^{\mathrm{g}}$ и $X^{\mathrm{c}}$, вошедшие в $(9)$, следует заменить на $x^{\mathrm{g}}$ и $\left.x^{\mathrm{c}} 1\right)$. Как обычно, условие нормировки имеет вид

$$
\frac{1}{a} \int d x^{\mathrm{g}} F_{10}\left(x^{\mathrm{g}}, t\right)=1,
$$

где $a$ - характерный пространственный параметр в $x$-направлении, рассчитанный на одну быструю частицу.

Уравнение Больцмана $(8),(9)$ линейно относительно $F_{10}$. Член, пропорциональный $\beta^{2}$, включает в себя двухчастичную функцию распределения $F_{02}$. Благодаря этому члену мы получаем совершенно логичный результат: при понижении температуры решетки величина интеграла столкновений (9) уменьшается так, что в пределе $T \rightarrow 0$ вклад столкновений в кинетический процесс обращается в нуль.

Стационарные функции решетки в (9) в квазигармоническом приближении равны

$$
\begin{aligned}
\widetilde{F}_{01}\left(q^{\mathrm{c}}\right) & =\sum_{m} w\left(\left|\mathbf{q}^{\mathrm{c}}-\mathbf{R}_{m}\right| ; \sigma_{T}^{2}\right), \\
\widetilde{F}_{02}\left(q_{1}^{\mathrm{c}}, q_{2}^{\mathrm{c}}\right) & =\sum_{\substack{m, n: \\
m \neq n}} w\left(\left|\mathbf{q}_{1}^{\mathrm{c}}-\mathbf{R}_{m}\right| ; \sigma_{T}^{2}\right) \cdot w\left(\left|\mathbf{q}_{2}^{\mathrm{c}}-\mathbf{R}_{n}\right| ; \sigma_{T}^{2}\right) .
\end{aligned}
$$

Здесь $\mathbf{R}_{m}$ - радиус-вектор $m$-го узла решетки, $w\left(q^{\mathrm{c}} ; \sigma_{T}^{2}\right)$ - нормальное распределение с дисперсией $\sigma_{T}^{2}$.

Итак, мы заменили систему уравнений (1), (2) для двух динамических подсистем уравнением (8), которое соответствует быстрой частице, движущейся в статическом термостате. Термостат лишен динамики, и в теории сохранились статические смещения $\sigma_{T}$ в $x$-направлении. Осталась одна динамическая система - быстрые частицы. Ранее переход к однокомпонентной системе выполнялся в теории смеси газов (см., например, [7]), что существенно упрощало исследование кинетических процессов. Эта же процедура облегчает решение нашей задачи. Дело в том, что больцмановское определение энтропии дается с точностью до постоянного слагаемого, если

\footnotetext{
1) В работе принята система единиц, в которой $\hbar=k_{\mathrm{B}}=1$.
} 
речь идет об однокомпонентной системе [8]. В качестве постоянной можно взять энтропию статической решетки [9]. Однако вид этой постоянной нам не потребуется, поскольку мы фокусируем внимание на источнике энтропии, а не на самой энтропии (см. ниже уравнение баланса энтропии).

\section{3. ВКЛАД СТОЛКНОВЕНИЙ В ЭНТРОПИЙНЫЙ ИСТОЧНИК И ВТОРОЕ НАЧАЛО ТЕРМОДИНАМИКИ}

Внесем некоторые дополнительные упрощения в член (9). Во-первых, будем считать, что распределение частиц в $x$-направлении является однородным, поскольку это отвечает физической ситуации плоскостного каналирования [5]. Во-вторых, учтем, что негармоничность потенциала плоскостного канала приводит к сглаживанию выражений в пространстве поперечных импульсов по мере роста глубины проникновения частиц в кристалл. Уравнение (8) применимо на глубинах, превышающих длину когерентности, поэтому допустимо усреднить столкновительный член по $p_{y}$. Наконец, учитывая, что в случае скользящих ударов легкой атомной частицы, движущейся в решетке из тяжелых атомов, величины $\zeta, \xi$ в (7) являются малыми, разложим столкновительный член по степеням этих величин и ограничимся старшими членами.

После введения указанных упрощений в (9) систему можно описать с помощью двух переменных: поперечной координаты $q_{x}$ и поперечного импульса $p_{x}$. Будем считать, что изменения $q_{x}$ и $p_{x}$ со временем являются достаточно медленными [4], [5]. Конкретные условия, налагаемые на переменные $q_{x}$ и $p_{x}$ с учетом корреляционного времени скоростей, корреляционного времени сил и т.п., а также на приращение поперечного импульса, можно найти в работе [10]. Как правило, эти условия дополняются условием малости пространственного градиента [11], чтобы исключить влияние вторичных эффектов (в частности, диффузии) на процесс каналирования. Что же касается градиента массовой скорости частиц, то в принятом приближении [6] он полностью отсутствует.

Интересно отметить, что практически таким же условиям удовлетворяют экстенсивные переменные в приближении крупнозернистого огрубления пространства и времени [12]. Такое описание первоначально использовалось только для макроскопических систем, однако гидродинамический подход можно применять и на микроскопическом уровне [13]. Для нас это обстоятельство представляется существенным, поскольку уравнение для плотности энтропии $\widetilde{S}\left(q_{x}, p_{x}, t\right)$, как правило [8], [12], записывается по аналогии с гидродинамическими уравнениями:

$$
\frac{\partial}{\partial t} \widetilde{S}\left(q_{x}, p_{x}, t\right)=-\nabla_{q_{x}} \tilde{J}_{S}\left(q_{x}, p_{x}, t\right)+\sigma_{S}\left(q_{x}, p_{x}, t\right),
$$

где $\tilde{J}_{S}\left(q_{x}, p_{x}, t\right)$ - плотность потока энтропии, $\sigma_{S}\left(q_{x}, p_{x}, t\right)$ - плотность энтропийного источника.

Мы рассматриваем силы с коротким радиусом действия. Известно [8], что в этом случае основной вклад в плотность энтропийного источника вносят динамические эффекты столкновений, тогда как вклад самосогласованного поля относительно мал. Поэтому плотность источника энтропии в пространственно неоднородной среде 
следует определить как полевую величину вида

$$
\sigma_{S}\left(q_{x}, p_{x}, t\right)=\frac{1}{a} \int_{-\infty}^{\infty} d q_{x}^{\prime} \int_{0}^{p_{x}} d p_{x}^{\prime}\left\{\frac{\partial}{\partial t} F_{10}\left(q_{x}^{\prime}, p_{x}^{\prime}, t\right)\right\}_{\mathrm{st}} \delta\left(q_{x}-q_{x}^{\prime}\right)
$$

Данное определение полностью соответствует физическому смыслу интенсивности источника, так как оно характеризует скорость изменения плотности энтропии в элементарном объеме за счет тех релаксационных процессов, которые приводят систему к состоянию локального квазиравновесия. Используя форму (11), мы можем обследовать каждую точку конфигурационного пространства в $x$-направлении с помощью дельта-функции, и если в точке $q_{x}$ нет быстрой частицы, то нет и вклада в энтропийный источник.

Подставляя выражение (9) в определение (11), находим, что плотность источника можно представить в виде суммы трех слагаемых:

$$
\sigma_{S}\left(q_{x}, p_{x}, t\right)=\sigma_{S}^{(1)}\left(q_{x}, p_{x}, t\right)+\sigma_{S}^{(2)}\left(q_{x}, p_{x}, t\right)+\sigma_{S}^{(3)}\left(q_{x}, p_{x}, t\right)
$$

Величина $\sigma_{S}^{(1)}$, записанная в симметризованном виде, равна

$$
\sigma_{S}^{(1)}\left(q_{x}, p_{x}, t\right)=\frac{1}{a} \int_{-\infty}^{\infty} d \lambda A\left(\lambda, q_{x}\right) I\left(q_{x}, p_{x}, t\right)
$$

где

$$
I\left(q_{x}, p_{x}, t\right)=\left|v_{x}^{\mathrm{g}}\right|\left(-\frac{\partial}{\partial E_{x}^{\mathrm{g}}} F_{10}\left(q_{x}, p_{x}, t\right)\right),
$$

$E_{x}^{\mathrm{g}}$ - поперечная энергия быстрой частицы,

$$
\begin{aligned}
A\left(\lambda, q_{x}\right) & =\frac{1}{v_{0}} \int d \mathbf{q}_{1}^{\mathrm{c}} \int d \mathbf{q}_{2}^{\mathrm{c}} G\left(-\mathbf{q}_{1}^{\mathrm{c}}+\frac{\lambda}{2}, \mathbf{q}_{2}^{\mathrm{c}}+\frac{\lambda}{2}, q_{x}\right), \\
G\left(q_{1}^{\mathrm{c}}, q_{2}^{\mathrm{c}}, q_{x}\right) & =g\left(q_{1}^{\mathrm{c}}, q_{2}^{\mathrm{c}}\right)\left\{\nabla_{q_{x}} \Phi\left(\left|\mathbf{q}^{\mathrm{g}}-\mathbf{q}_{1}^{\mathrm{c}}\right|\right)\right\} \nabla_{q_{x}} \Phi\left(\left|\mathbf{q}^{\mathrm{g}}-\mathbf{q}_{2}^{\mathrm{c}}\right|\right) .
\end{aligned}
$$

Парная корреляционная функция кристалла, вошедшая в $G\left(q_{1}^{\mathrm{c}}, q_{2}^{\mathrm{c}}, q_{x}\right)$, имеет вид

$$
g\left(q_{1}^{\mathrm{c}}, q_{2}^{\mathrm{c}}\right)=\widetilde{F}_{02}\left(q_{1}^{\mathrm{c}}, q_{2}^{\mathrm{c}}\right)-\widetilde{F}_{01}\left(\left|\mathbf{q}_{1}^{\mathrm{c}}\right|\right) \widetilde{F}_{01}\left(\left|\mathbf{q}_{2}^{\mathrm{c}}\right|\right) .
$$

Величины $\sigma_{S}^{(2)}$ и $\sigma_{S}^{(3)}$ равны

$$
\begin{aligned}
\sigma_{S}^{(2)}\left(q_{x}, p_{x}, t\right)=- & \frac{1}{a M} \int d q_{1}^{\mathrm{c}} \widetilde{F}_{01}\left(q_{1}^{\mathrm{c}}\right) \nabla_{q_{x}}^{2} \Phi\left(\left|\mathbf{q}^{\mathrm{g}}-\mathbf{q}_{1}^{\mathrm{c}}\right|\right) \times \\
& \times \int_{0}^{\infty} d \tau \tau \nabla_{q_{x}} \Phi\left(\left|\mathbf{q}^{\mathrm{g}}-\mathbf{q}_{1}^{\mathrm{c}}-\left(\mathbf{v}^{\mathrm{g}}-\mathbf{v}_{1}^{\mathbf{c}}\right) \tau\right|\right) F_{10}\left(q_{x}, p_{x}, t\right), \\
\sigma_{S}^{(3)}\left(q_{x}, p_{x}, t\right)=\frac{2}{a} & \int d q_{1}^{\mathrm{c}} \widetilde{F}_{01}\left(q_{1}^{\mathrm{c}}\right) \nabla_{q_{x}} \Phi\left(\left|\mathbf{q}^{\mathrm{g}}-\mathbf{q}_{1}^{\mathrm{c}}\right|\right) \times \\
& \times \int_{0}^{\infty} d \tau \tau \nabla_{q_{x}} \Phi\left(\left|\mathbf{q}^{\mathrm{g}}-\mathbf{q}_{1}^{\mathrm{c}}-\left(\mathbf{v}^{\mathrm{g}}-\mathbf{v}_{1}^{\mathrm{c}}\right) \tau\right|\right) \nabla_{q_{x}} F_{10}\left(q_{x}, p_{x}, t\right) .
\end{aligned}
$$


Как следует из $(13)$, функция $K^{(1)}\left(q_{x}\right)$ обратно пропорциональна скорости частиц, $K^{(1)}\left(q_{x}\right) \sim 1 / v_{0}$. Выполнив преобразования в $(14)$, находим

$$
\sigma_{S}^{(2)} \sim \frac{1}{v_{0}^{2}}, \quad \sigma_{S}^{(3)} \sim \frac{1}{v_{0}^{2}}
$$

Поэтому в случае высоких скоростей, точнее для частиц с энергией $E_{0} \sim 1$ МэВ, в выражении (12) достаточно ограничиться первым членом $\sigma_{S}^{(1)}$.

Далее, подставляя функции $\widetilde{F}_{01}\left(q^{\mathrm{c}}\right)$ и $\widetilde{F}_{02}\left(q_{1}^{\mathrm{c}}, q_{2}^{\mathrm{c}}\right)(10)$ в выражение $(13)$, находим

$$
\begin{gathered}
\int_{-\infty}^{\infty} d \lambda A\left(\lambda, q_{x}\right)=K\left(q_{x}\right) \equiv K^{(1)}\left(q_{x}\right)-\Delta K^{(1)}\left(q_{x}\right), \\
K^{(1)}\left(q_{x}\right)=2 n_{\mathrm{c}} \cdot 2 l \sum_{n} B_{n} \int d q^{\mathrm{c}} e^{i \pi n q_{x}^{\mathrm{c}} / l} \nabla_{q_{x}} \widetilde{\Phi}\left(\left|\mathbf{q}-\mathbf{q}^{\mathrm{c}}\right|\right) \times \\
\quad \times \int_{0}^{\infty} d \tau \nabla_{q_{x}} \widetilde{\Phi}\left(\left|\mathbf{q}-\mathbf{q}^{\mathrm{c}}-\mathbf{v}_{0} \tau\right|\right), \\
\Delta K^{(1)}\left(q_{x}\right)=2 n_{\mathrm{c}} \cdot 2 l \sum_{n} B_{n}^{2} \int d q_{1}^{\mathrm{c}} \int d q_{2}^{\mathrm{c}} e^{\left(i \pi n\left(q_{1 x}^{\mathrm{c}}+q_{2 x}^{\mathrm{c}}\right) / l\right) \widetilde{n}_{G}\left(\rho_{1}^{\mathrm{c}}-\rho_{2}^{\mathrm{c}}\right)} \times \\
\times \nabla_{q_{x}} \widetilde{\Phi}\left(\left|\mathbf{q}-\mathbf{q}_{1}^{\mathrm{c}}\right|\right) \int_{0}^{\infty} d \tau \nabla_{q_{x}} \widetilde{\Phi}\left(\left|\mathbf{q}-\mathbf{q}_{2}^{\mathrm{c}}-\mathbf{v}_{0} \tau\right|\right),
\end{gathered}
$$

где

$$
B_{n}=\frac{1}{2 l} \exp \left[-\left(\frac{\pi n}{l}\right)^{2} \frac{\sigma_{\mathrm{T}}^{2}}{2}\right], \quad \tilde{n}_{\mathrm{G}}(\rho)=\frac{1}{2 \pi 2 \sigma_{\mathrm{T}}^{2}} e^{-\rho^{2} /\left(2 \cdot 2 \sigma_{\mathrm{T}}^{2}\right)},
$$

$\mathbf{q}^{\mathrm{c}}=\left\{q_{x}^{\mathrm{c}}, \rho^{\mathrm{c}}\right\}, l-$ полуширина плоскостного канала, $n=0, \pm 1, \pm 2, \ldots$.

Принимая в качестве $\Phi$ экранированный кулоновский потенциал с радиусом эффективного действия $a_{0}$, преобразуем основной член (15б) к виду, удобному для численного интегрирования. В результате находим

$$
\begin{aligned}
& K^{(1)}\left(q_{x}^{\mathrm{g}}\right)=\frac{1}{v_{0}}\left(Z_{1} Z_{2} e^{2}\right)^{2} \sum_{h_{n}} B^{(1)}\left(h_{n}\right) P\left(h_{n}\right) \cos \left(h_{n} q_{x}^{\mathrm{g}}\right), \\
& B^{(1)}\left(h_{n}\right)=\int_{0}^{\Lambda_{1}} d k k^{3} \frac{1}{\beta_{n}(k)\left[\beta_{n}(k)+\eta_{n}(k)\right]},
\end{aligned}
$$

где $h_{n} \equiv h_{x}-(2 \pi / 2 l) n-$ вектор обратной решетки, перпендикулярный плоскости канала,

$$
\begin{aligned}
P\left(h_{n}\right) & =2 \pi n_{\mathrm{c}} e^{-h_{n}^{2} \sigma_{\mathrm{T}}^{2} / 2}, & \beta_{n}(k) & =\left(\eta_{n}^{2}(k)-4 k^{2} h_{n}^{2}\right)^{1 / 2}, \\
\eta_{n}(k) & =k^{2}+h_{n}^{2}+\left(\frac{1}{a_{0}}\right)^{2}, & \Lambda_{1} & =\left(Z_{1} Z_{2} e^{2}\right) \frac{1}{E_{0}} \frac{M}{M+m} .
\end{aligned}
$$

Окончательное выражение для величины $\Delta K^{(1)}\left(q_{x}\right)(15$ в), характеризующей вклад корреляционных эффектов, имеет очень громоздкий вид. Поэтому корреляционная поправка $\Delta K^{(1)}\left(q_{x}\right)$ показана только графически (см. рисунок). 


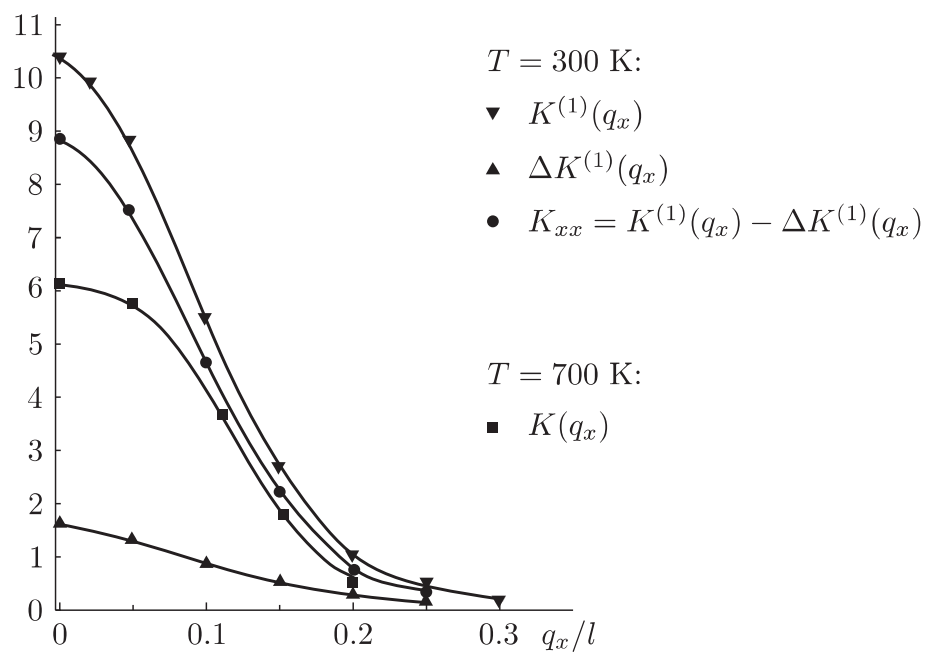

Величина $K\left(q_{x}\right)$, заданная формулами (15), представляет собой диагональный элемент корреляционной матрицы сил, действующих на частицу со стороны атомов решетки.

Результаты численного интегрирования выражений (16), (15в) представлены на рисунке. Расчеты выполнены для ионов гелия с энергией $1.5 \mathrm{MэВ,} \mathrm{движущихся}$ в плоскостном канале (100) кристалла германия, при двух температурах $\mathrm{T}=300 \mathrm{~K}$ и $\mathrm{T}=700 \mathrm{~K}$. Как показано на рисунке, $K^{(1)}\left(q_{x}\right), \Delta K^{(1)}\left(q_{x}\right)$ и $K\left(q_{x}\right)$ имеют вид гауссовских функций (на рисунке представлена только правая часть этих функций), причем основная часть матрицы сил $K^{(1)}\left(q_{x}\right)$ является положительной, а корреляционная часть, которая во все выражения входит со знаком минус, дает отрицательную поправку. Однако разность величин $K^{(1)}\left(q_{x}\right)-\Delta K^{(1)}\left(q_{x}\right)$ положительна. Принимая во внимание тот факт, что $K\left(q_{x}\right) \geqslant 0$ и $I\left(q_{x}, p_{x}, t\right)>0$, приходим к заключению, что в случае высокоэнергетических каналированных частиц выполняется условие

$$
\sigma_{S}\left(q_{x}, p_{x}, t\right)=\frac{1}{a} K\left(q_{x}, p_{x}, t\right) I\left(q_{x}, p_{x}, t\right) \geqslant 0 .
$$

Положительный знак интенсивности источника энтропии (17), которая характеризует локальное производство энтропии в единицу времени, и есть не что иное, как формулировка второго закона термодинамики. Эволюция закрытой неравновесной подсистемы сопровождается ростом ее энтропии, так что при достижении термодинамического равновесия энтропия достигает максимального значения. Спонтанный переход к равновесному значению обеспечивается за счет механизма рассеяния, в частности за счет рассеяния каналированных частиц на тепловых отклонениях атомов решетки, при этом величина энтропийного источника (11) определяется столкновительным членом (9), вошедшим в кинетическое уравнение (8).

Второй закон выполняется только для закрытых подсистем, к числу таких подсистем относятся и частицы, которые движутся в кристалле в режиме каналирования, при этом следует подчеркнуть, что мы имеем в виду только энтропию рассматриваемой подсистемы, поскольку в общем необратимом процессе может также иметь место возрастание энтропии термостата [9]. 


\section{Список литературы}

[1] Н. Н. Боголюбов, Проблемы динамической теории в статистической физике, ГИТГЛ, M.-Л., 1946.

[2] П. П. Кулик, "Упругие взаимодействия и явления переноса", Очерки физики и химии низкотемпературной плазмы, ред. Л. С. Полак, Наука, М., 1971, 5-55.

[3] Yu. A. Kashlev, N. M. Sadykov, Phys. Stat. Sol. (b), 197:1 (1996), 19-29.

[4] Й. Линдхард, УФН, 99:2 (1969), 249-296.

[5] D. S. Gemmell, Rev. Modern Phys., 46:1 (1974), 129-227.

[6] M. T. Robinson, Phys. Rev., 179:2 (1969), 327-342.

[7] E. Moreau, J. Salmon, J. Phys. Radium, 21 (I960), 217-222.

[8] Р. Балеску, Равновесная и неравновесная статистическая механика, Т. 2, Мир, М., 1978.

[9] Р. Кубо, Термодинамика, Мир, М., 1970.

[10] Ю. А. Кашлев, ТМФ, 140:1 (2004), 86-99.

[11] T. Oshiyama, H. Kudo, M. Mannami, J. Phys. Soc. Japan, 36:4 (1974), 1107-1113.

[12] Дж. Кайзер, Статистическая термодинамика неравновесных прочессов, Мир, М., 1990.

[13] M. S. Green, J. Chem. Phys., 20:8 (1952), 1281-1295.

Поступила в редакцию 29.04.2008, после доработки 15.05.2009 